\title{
Residentes de enfermagem em uma unidade de internação pediátrica: relato de experiência do processo de enfermagem
}

\author{
Nursing residents in a pediatric inpatient unit: experience report of the nursing process \\ Residentes de enfermería en una unidad de internación pediátrica: relato de experiencia \\ del proceso de enfermería
}

Jonathan Douglas Pinheiro Sampaio ${ }^{1 *}$, Andressa Tavares Parente ${ }^{1}$, Hilma Solange Lopes Souza ${ }^{1}$, Cintia Yolette Urbano Pauxis Aben-Athar Valentim ${ }^{1}$, Francisca Elissandra Ribeiro dos Santos ${ }^{1}$, Marléa Guimarães Palheta ${ }^{1}$, Alana Celeste Campos Dias ${ }^{1}$, Danielle Cardoso Portilho ${ }^{1}$, Mayara Nicodemos da Conceição ${ }^{1}$, Angeline do Nascimento Parente ${ }^{1}$.

\section{RESUMO}

Objetivo: Compartilhar a experiência vivenciada, por enfermeiros residentes, em uma clínica pediátrica de um hospital universitário, no contexto da implementação da Sistematização da Assistência de Enfermagem. Relato de experiência: Estudo descritivo, de abordagem qualitativa na modalidade de relato de experiência, realizado em julho a agosto de 2019, durante a vivência da prática profissional de residentes de enfermagem do Programa de Residência Multiprofissional em Atenção à Clínica Integrada, em uma unidade de internação pediátrica de um hospital. Na experiência, foi possível compreender o processo de trabalho e identificar o modo como à assistência de enfermagem estava sendo realizada junto ao paciente pediátrico e seu acompanhante. Foram realizadas atividades gerenciais e assistências pelos residentes, supervisionadas pelo preceptor, que estimulou a desenvolverem um pensamento crítico reflexivo e tomar decisões perante os problemas encontrados. Considerações finais: A vivência dos residentes na unidade de internação trouxe reflexões e delineamentos sobre o processo de trabalho do enfermeiro, a especificidade da assistência pautada na criança e sua família, o que contribuiu diretamente com a qualificação da assistência e a preservação da segurança do paciente pediátrico.

Palavras-chave: Pediatria, Cuidados de enfermagem, Processo de enfermagem.

\begin{abstract}
Objective: To share the experience lived by resident nurses, in a pediatric clinic of a university hospital, during the implementation of the Nursing Care Systematization. Experience report: It was developed a descriptive study, with qualitative approach in the form of an experience report, conducted from July to August 2019, during the professional practice of nursing residents in the Multiprofessional Residency Program in Integrated Clinical Care, in a Pediatric Inpatient Unit. Through the experience, it was possible to understand the work process and to identify how nursing care was being performed with the pediatric patients and their families/caregivers. Management activities and assistance were carried out by residents, supervised by the preceptor, who encouraged them to develop reflective critical thinking and make decisions when problems emerged. Final considerations: The residents' experience in the Inpatient Pediatric Unit brought reflections and outlines about the nurse's work process, the specificity of assistance focused on the children and their families, which directly contributed to the quality of care and the preservation of pediatric patient safety.
\end{abstract}

Keywords: Pediatrics, Nursing care, Nursing process.

\section{RESUMEN}

Objetivo: Compartir la experiencia vivida, por los enfermeros residentes, en una clínica pediátrica de un hospital universitario, en el contexto de la implementación de la Sistematización de la Asistencia de Enfermería. Relato de experiencia: Estudio descriptivo, de abordaje cualitativa en la modalidad de relato

1 Universidade Federal do Pará (UFPA), Belém - PA. *E-mail: doug.maia2013@hotmail.com 
de experiencia, realizado en julio a agosto de 2019, durante la vivencia de la práctica profesional de residentes de enfermería del Programa de Residencia Multiprofesional en Atención a la Clínica Integrada, en una unidad de internación pediátrica de un hospital. En la experiencia se pudo entender el proceso de trabajo e identificar cómo se realizaban los cuidados de enfermería con el paciente pediátrico y su acompañante. Las actividades de gestión y asistencia fueron realizadas por los residentes, supervisados por el preceptor, que les estimuló a desarrollar un pensamiento crítico reflexivo y a tomar decisiones ante los problemas encontrados. Consideraciones finales: La experiencia de los residentes en la unidad de internación llevó a reflexiones y delineamientos sobre el proceso de trabajo de enfermería y la especificidad de los cuidados basados en los niños y sus familias, lo que contribuyó directamente para la calidad de los cuidados y la preservación de la seguridad del paciente pediátrico.

Palabras clave: Pediatría, Cuidados de enfermería, Proceso de enfermería.

\section{INTRODUÇÃO}

A Residência em Enfermagem objetiva o treinamento em serviço, de forma a articular o conhecimento teórico e a ação, qualificando profissionais a partir da realidade prática, visto que estão diretamente em contato com o cotidiano do trabalho em saúde. O enfermeiro residente deve promover o gerenciamento do cuidado de forma interdisciplinar, buscando a integralidade da assistência em sua área de concentração e contribuindo para uma articulação com os demais serviços que compõe a rede de atenção à saúde (SOARES RSA, et al., 2017).

A Sistematização da Assistência de Enfermagem (SAE) viabiliza atender às necessidades individuais dos clientes, pois organizar o trabalho da equipe de enfermagem e proporciona maior eficácia na assistência, tornando-se uma ferramenta que eleva a qualidade do serviço. Inclui uma série de ações planejadas destinadas a alcançar o bem-estar ou a mais alta qualidade de vida pelo maior tempo possível, objetivando tornar o conhecimento e o fazer da enfermagem uma profissão por meio de uma linguagem padronizada e universal (TANNURE MC e PINHEIRO AM, 2019).

O enfermeiro, ao implementar a SAE, deve ser capaz de planejar as ações da equipe de enfermagem, avaliar suas intervenções e alcançar a integralidade do cuidado humanizado (TANNURE MC e PINHEIRO AM, 2019). O cuidado de enfermagem à criança e ao adolescente não deve incluir apenas o cuidado físico / biológico, mas deve considerar suas particularidades, ou seja, suas necessidades emocionais, sociais e espirituais, a partir do momento em que o enfermeiro tem conhecimento sobre os níveis de necessidades de sua clientela específica, os diagnósticos, resultados e as intervenções terão melhor resolução e qualidade (FALKE ACS, et al., 2014).

Nesse cenário, ressalta-se a importância da assistência de enfermagem centrada na criança e na família, estabelecido através da transmissão de orientações educativas, cuidados direcionados à criança hospitalizada e familiares, além das ações técnicas destes profissionais de saúde, com foco no cuidado integral que possibilite conhecer os diversos aspectos que são inerentes à vivência da criança e família no processo de hospitalização (AZEVÊDO AVS, et al., 2017).

Diante do exposto, o objetivo deste presente estudo é de compartilhar a experiência vivenciada por residentes de enfermagem em uma clínica pediátrica de um hospital universitário, no contexto da implementação da Sistematização da Assistência de Enfermagem.

\section{RELATO DE EXPERIÊNCIA}

Estudo descritivo de abordagem qualitativa na modalidade de relato de experiência, que emergiu da vivência da prática profissional a partir da experiência como enfermeiros residentes do Programa de Residência Multiprofissional em Atenção à Clínica Integrada, em uma unidade de internação pediátrica de um hospital universitário.

O cenário da experiência constitui-se de 25 leitos, para receber internações de crianças de 29 dias até 12anos, para tratamento de doenças infectocontagiosas, neoplásicas e/ou crônico-degenerativas. A atuação 
na clínica ocorreu no período de julho a agosto de 2019. A equipe de enfermagem da pediatria é composta por 25 técnicos de enfermagem, 12 auxiliares de enfermagem, 7enfermeiros que se dedicam à assistência às crianças internadas, uma enfermeira que coordena o cuidado e duas enfermeiras que atuam em atividades administrativas.

Ao iniciar a prática, a coordenação de enfermagem da pediatria recepcionou os residentes para realizar a integração com a equipe, onde foram apresentados as normas, rotinas, protocolo e organograma de funcionamento da unidade. Após acolhimento no setor, os residentes vivenciaram um período de adaptação ao campo de atuação, devido à complexidade, dinamicidade e às particularidades do processo de trabalho em uma clínica pediátrica.

A rotina iniciava-se com a passagem do plantão entre os enfermeiros responsáveis e em seguida era realizada a divisão de escala dos técnicos de enfermagem do turno. Na sequência, realizava-se a visita de enfermagem e a avaliação geral do estado do paciente com uso de instrumento, contendo informações necessárias para classificá-lo para o nível de assistência.

Foram também realizadas análise dos prontuários, admissão, exame físico, entrevistas, histórico do paciente, evolução e registro, discussão de casos clínicos, construção de planos individuais de cuidado enfermagem, curativos, avaliação do risco de queda, orientações às crianças e acompanhante, implementação da sistematização, entre outros. Todas as atividades foram supervisionadas pelo preceptor.

No que concerne a presença do acompanhante, foi visto que a existência da mãe era predominante, onde ela participava ativamente no processo de hospitalização, pois contribuía para a recuperação, tratamento e suporte emocional do filho; e também para o desenvolvimento de outras funções, as quais incluíam: auxiliar no cuidado e acompanhamento quanto a assistência recebida.

Durante a experiência na clínica foi possível compreender o processo de trabalho e identificar o modo como à assistência de enfermagem estava sendo produzida junto ao paciente pediátrico e seu acompanhante.

$\mathrm{Na}$ Unidade pediátrica foram utilizadas cinco etapas no Processo de Enfermagem (PE), interrelacionadas, recorrentes e interdependentes, discutidas pela Resolução COFEN no 358/2009, assim definidas: Histórico de enfermagem (HE), Diagnóstico de Enfermagem (DE), Planejamento, Implementação de Enfermagem (IE) e Avaliação (BRASIL, 2009).

Seguindo essas etapas, quando admitida, a criança era recepcionada pela equipe, e assim direcionada para o leito. Posteriormente, com a finalidade de levantar os dados sobre as condições de vida e saúde do paciente e da família, os residentes e o preceptor realizavam entrevista, anamnese e o exame físico.

Nesta primeira fase, foi utilizado um instrumento de coleta de dados do HE, que propiciou o levantamento de informações e tornou possível a identificação, análise e avaliação dos problemas apresentados pelas crianças. Este instrumento foi construído em conformidade com a teoria das Necessidades Humanas Básicas (NHB) de Wanda de Aguiar Horta; era composto por dados de internação, queixas, história da doença atual, avaliação cognitiva, antecedentes pessoais e familiares, hábitos de vida, situação socioeconômica e exame físico.

Uma vez realizada a investigação, os residentes e o preceptor identificavam os problemas mais comuns, as necessidades e o grau de dependência da criança, definindo os DE. O instrumento utilizado para estabelecer os DE foi pautado na taxonomia da Associação Norte Americana de Diagnósticos de Enfermagem (NANDA-I). Neste instrumento constava uma lista de DE, assim como os fatores relacionados.

Em seguida, a partir dos achados das NHB, caracterizou-se o Planejamento, onde foram determinadas as necessidades prioritárias dos pacientes, de forma sistematizada e fornecido à criança frente aos diagnósticos estabelecidos.

Posteriormente, delimitou-se a IE, descrevendo intervenções cotidianas, com foco na coordenação das ações da equipe de enfermagem e na execução dos cuidados e assistência específicos da criança, dando 
continuidade e manutenção do cuidado e avaliação no dia a dia. Baseado na Classificação das Intervenções de Enfermagem (NIC) dispõem-se as Prescrições de Enfermagem, atrelado no instrumento dos Diagnósticos, onde era feito o aprazamento dessa prescrição.

Da mesma forma, este instrumento constava de uma lista de intervenções definidas, com os cuidados frequentes fundamentados nos diagnósticos de enfermagem recorrentes. O enfermeiro sinalizava as intervenções necessárias e delimitava os horários que deveriam ser realizadas pelos enfermeiros ou técnicos de enfermagem. Se necessário, poderia acrescentar outras intervenções não previstas na lista. $O$ impresso da SAE era reavaliado a cada 24 horas.

A última etapa era a Avaliação do cuidado, concentrada em analisar se os resultados esperados foram alcançados com as intervenções aplicadas. Os residentes ficavam responsáveis de checar se os cuidados prestados eram realizados, conforme prescrito. Percebeu-se baixa adesão a checagem da prescrição de enfermagem, mesmo quando o cuidado havia sido prestado. Em seguida efetuavam a evolução de após a avaliação do estado geral do paciente.

Nos aspectos do cuidado, contemplou-se ainda o uso do Sistema de Classificação de Paciente FUGULIN que permitia o acompanhamento e avaliação diária pela Enfermagem do perfil dos pacientes internados e a escala de Braden para classificar o risco da criança em desenvolver Lesão por Pressão (LP) (FUGULIN FMT, et al., 2005; BRADEN B e BERGSTROM, 1996).

Com a finalidade de classificar o paciente quanto ao grau de dependência em relação a enfermagem, 0 enfermeiro utilizava a Escala de FUGULIN, que preenchia a ficha conforme os dados coletados no exame físico, utilizando a graduação de 1 a 4, de acordo com a área do cuidado, dividido em 12 itens: estado mental, oxigenação, sinais vitais, motilidade, deambulação, alimentação, cuidado corporal, eliminação, terapêutica, comprometimento tecidual, curativo e tempo de curativos. Após isso, era somado o escore nas suas respectivas áreas de cuidado no impresso e anotar o valor total; conforme o valor somatório, era classificado o tipo de cuidado: cuidado como mínimo (09-14 pontos), intermediário (15-20 pontos), alta dependência (21-26 pontos), semi-intensivo (27-31 pontos) e cuidados intensivo (acima de 31 pontos) (FUGULIN FMT, et al., 2005).

O perfil de assistência de enfermagem, predominantemente, requeria cuidados intermediários, não raro apresentando necessidades de alta dependência e/ou cuidados semi-intensivos. Durante o exame físico os residentes eram responsáveis por supervisionar o risco da criança em desenvolver LP e para isso utilizava a escala de Braden, proporcionando uma avaliação mais ampla, que considerava a intensidade e a duração da pressão por meio da avaliação da percepção sensorial, a umidade, atividade, mobilidade, nutrição, friç̧ão e cisalhamento. A criança era classificada conforme o escore: sem risco, risco leve, intermediário e alto (BRADEN B e BERGSTROM, 1996).

Quanto às barreiras encontradas para a operacionalização da SAE, foi identificado a sobrecarga de demandas dos enfermeiros em realizar o processo de cuidado, a adesão parcial ao plano de cuidados traçado, por parte da equipe de técnicos de enfermagem, que muitas vezes não era implementado, uma vez que os resultados indicavam a ausência de preenchimento/checagem dos cuidados.

Ainda, observou-se que os instrumentos do PE utilizados na unidade pediátrica apresentavam limitações quanto ao plano de cuidados às crianças, pois os $\mathrm{DE}$ e as intervenções de enfermagem implementados não considerava as peculiaridades desses indivíduos.

\section{DISCUSSÃO}

As experiências adquiridas durante o campo de prática colaboram para os residentes descobrirem habilidades profissionais e assumirem responsabilidades, que são designadas durante a prática e se tornarão essenciais à sua futura formação (EVANGELISTA DL e IVO OP, 2014).

Segundo Negreiros RV e Lima VC (2018), a prática em campo hospitalar propicia ao residente de enfermagem o contato diário das situações reais da sua profissão. A utilização da Sistematização da 
Assistência de Enfermagem como ferramenta de trabalho, também tem demonstrado ser relevante para a atuação, pois ela tem o intuito de identificar as necessidades dos pacientes e propor cuidados necessários que deverão ser realizados pela equipe.

Na pediatria, os pacientes apresentam maior grau de dependência de cuidados e a hospitalização causa impactos negativos na vida dessa população. Diante disto, evidencia-se a importância da assistência de enfermagem centrada na criança e na família, dado que o cuidado prestado pela equipe de saúde à criança requer observação e cuidados durante seu planejamento e execução (FALKE ACS, et al., 2014).

Pesquisas sobre acompanhantes apontam mulheres, especialmente mães, como as únicas ou principais cuidadoras de familiares doentes, sejam filhos ou não (PEREIRA LP e GUEDES MVC, 2011). Deste modo, ao desempenhar o papel de cuidadora no contexto da hospitalização infantil, a mãe se configura como agente promotora que facilita o trabalho do profissional de saúde (LIMA RM, et al., 2019).

Considerando as vulnerabilidades do paciente pediátrico, ao aplicar a SAE, o enfermeiro organiza e qualifica o seu trabalho, proporcionando mais segurança para os mesmos. Portanto, considera-se a implantação da SAE, um instrumento de comunicação que efetiva as atividades do enfermeiro para assistir a criança, promovendo subsídios para o planejamento, coordenação e avaliação das ações de enfermagem (TANNURE MC e PINHEIRO AM, 2019).

Segundo NANDA (2013), o diagnóstico de enfermagem exige competências nos domínios intelectual, interpessoal e técnico e é caracterizado como julgamentos clínicos sobre as respostas do indivíduo, da família ou da comunidade. Os diagnósticos de enfermagem previamente definidos na clínica pediátrica deste estudo diferenciam-se de uma pesquisa realizada em um hospital público em Campina Grande-PB, onde foram identificados 10 diagnósticos de enfermagem mais frequentes em pacientes internados em uma unidade pediátrica (GUEDES DM, et al., 2015).

$\mathrm{Na}$ experiência verificou-se a baixa adesão da equipe de enfermagem ao formulário da SAE. Além disso, o instrumento utilizado na execução da SAE apresentava limitações quanto ao plano de cuidados, onde os DE e IE eram predefinidos e não consideravam as peculiaridades dos pacientes, fazendo com que a assistência fosse realizada de forma rotineira e mecanizada.

Estudos enfatizam que a SAE feita de forma repetitiva, limitando-se a cuidados rotineiros, não respeita a individualidade do paciente. Sendo assim, é necessário estimular o raciocínio clínico do enfermeiro para que a particularidade do paciente possa ser considerada e o cuidado seja efetuado de forma eficaz (TAVARES TS, et al., 2013).

A utilização da Escala de Avaliação de Risco de desenvolvimento de LP fornece um plano sistemático para cuidado ao paciente pediátrico, o que contribui para o diagnóstico, tratamento e prevenção dessas lesões (SOARES PO, et al., 2015). A escala de Braden é uma das ferramentas para auxiliar na detecção do risco LPP, além de possibilitar ao profissional de enfermagem um melhor delineamento das prescrições de cuidados que devem ser fornecidos a esses pacientes (DINIZ SMO, et al., 2017).

A classificação de pacientes pediátricos como uma ferramenta para determinar as necessidades de cuidados, pode fornecer um método eficaz para equilibrar as necessidades, a oferta e a qualidade das unidades de internação pediátrica. Por meio da dimensão pessoal, o enfermeiro pode identificar o perfil do paciente sobre a complexidade assistencial, determinar o tempo de atendimento de acordo com a categoria profissional, calcular o percentual de ausências planejadas e não planejadas da equipe de enfermagem e determinar a jornada efetiva de trabalho (MARTINS FVS, 2017).

A não realização da SAE também emerge devido à falta de obrigatoriedade no serviço e da ausência de treinamento. Segundo Martins VF, et al. (2013), as dificuldades para operacionalizar a SAE envolvem fatores conceituais, estruturais e organizacionais. Os recursos humanos são os mais relevantes para a operacionalização desta ferramenta.

Durante a experiência foi oportunizado conhecer e vivenciar a rotina do setor e realizar diversas atividades gerenciais e assistências de sua competência. Diante das especificidades e complexidades da 
pediatria, os conhecimentos adquiridos tornam-se fundamentais, pois possibilitam a ampliação de habilidades, raciocínio crítico, liderança e tomada de decisão frente à cuidados de crianças hospitalizadas, fazendo com que os residentes desenvolvam uma postura ética profissional, aprendendo a criar o vínculo com o indivíduo e suas famílias, para que se possa estabelecer uma relação de confiança e comprometimento.

Dado o exposto, conclui-se que a vivência na unidade pediátrica pelos residentes fez com que os mesmos refletissem, compreendessem e buscassem métodos que colaborasse para o processo de trabalho do enfermeiro, e assim contribuindo diretamente com a qualificação da assistência e a preservação da segurança do paciente pediátrico. Além do mais, perceberem a importância do PE como elemento modificador do processo de trabalho do enfermeiro, e suas dimensões de cuidado, proporcionando uma assistência segura e de qualidade à criança internada, pois a partir da realização de suas etapas, o cuidado oferecido torna-se mais adequado.

\section{REFERÊNCIAS}

1. AZEVÊDO AVS, et al. Interação equipe de enfermagem, família, e criança hospitalizada: revisão integrativa. Ciênc. Saúde Coletiva, Rio de Janeiro, 2017; 22(11): 3653-3666.

2. BRADEN B, BERGSTROM N. A conceptual schema for the study of the etiology of pressure sore. Rehabilitation Nursing, 1996.

3. BRASIL. Conselho Federal de Enfermagem (COFEN). RESOLUÇÃO COFEN-358 de 15 out 2009. Dispõe sobre a Sistematização da Assistência de Enfermagem e a implementação do Processo de Enfermagem em ambientes, públicos ou privados, em que ocorre o cuidado profissional de Enfermagem, e dá outras providências. 2009. Disponível em: http://www.cofen.gov.br/resoluo-cofen-3582009_4384.html.

4. DEMARCO EA. Formação multiprofissional como tecnologia para qualificar a Atenção Primária à Saúde no SUS: avaliação de um programa de residência [dissertação]. Porto Alegre: Universidade Federal do Rio Grande do Sul; 2011.

5. NORTH AMERICAN NURSING DIAGNOSIS ASSOCIATION (NANDA). Diagnósticos de enfermagem da Nanda: definições e classificação 2009-2011. Porto Alegre: Artmed, 2013.

6. DINIZ SMO, et al. Prevalência de Úlcera por Pressão em Unidade de Terapia Intensiva em Hospitais Públicos. In: Anais do I international nursing congress Theme: Good practices of nursing representations in the construction of society [Internet]. Brasil, Espanha, 2017.

7. EVANGELISTA DL, IVO OP. Contribuições do estágio supervisionado para a formação do profissional de enfermagem: expectativas e desafios. RevEnfermContemp, 2014; 3(2): 123-30.

8. FALKE ACS, et al. Estratégias utilizadas pelos profissionais da enfermagem na abordagem a crianças hospitalizadas. Revista contexto e saúde, 2014; 18(34): 9-14.

9. FUGULIN FMT, et al. Sistema de classificação de pacientes: identificação do perfil assistencial dos pacientes das unidades de internação do HU-USP. Rev Latino-AmEnferm. 2005; 13(1): 72-8.

10. GUEDES DM, et al. Diagnósticos de enfermagem mais frequentes em uma unidade de terapia. RevEnferm UFSM, $2015 ; 5(3): 476-485$.

11. LIMA RM, et al. Experiências de Mães Durante a Internação Hospitalar de Seus Filhos. J. res.: fundam. care. online, 2019; 11(5): 1286-1292.

12. MARTINS FVS. Sistema de classificação de pacientes em enfermagem na pediatria de um hospital de ensino. Trabalho de Conclusão de Curso. Universidade Federal de Mato Grosso do Sul, 2017.

13. MARTINS VF, et al. A viabilidade da sistematização da assistência de enfermagem em serviço pediátrico: uma abordagem reflexiva. Revista Eletrônica Gestão \& Saúde, 2013; 4(1): 1820-1834.

14. NEGREIROS RV, LIMA VC. Importância do estágio supervisionado para o Acadêmico de Enfermagem no Hospital: Compartilhando experiências vivenciadas com a equipe de Trabalho. Revista da Universidade Vale do Rio Verde, 2018; 16(2): 1-7.

15. PEREIRA LP, GUEDES MVC. Dificuldades de mães de adolescentes diabéticos tipo 1 no acesso ao atendimento de saúde. Revrene, 2011; 12(3): 487-93.

16. SOARES PO, et al. Uso da escala de Braden e caracterização das úlceras por pressão em acamados hospitalizados. RevEnfermUFPI, 2015; 4(3): 18-23.

17. SOARES RSA, et al. Vivências de residentes enfermeiros no programa de residência multiprofissional em saúde. Santa Maria, 2017; 43(1): 13-21.

18. TANNURE MC, PINHEIRO AM. SAE. Sistematização da Assistência de Enfermagem. Guia Prático 3a. ed. Rio de Janeiro: Guanabara Koogan, 2019.

19. TAVARES TS, et al. Avaliação da implantação da sistematização da assistência de enfermagem em uma unidade pediátrica. REME Rev Min Enferm, 2013 abr/jun; 17(2): 278-286. 